\title{
Endovascular Treatment for Intracranial Aneurysms: A Nationwide Survey in Korea
}

\author{
Jung-Jae Kim, $\mathrm{MD}^{1}$, Kwang-Chun Cho, $\mathrm{MD}^{2}$, Woo Sang Jung, MD, PhD³, Sang Hyun Suh, MD, $\mathrm{PhD}^{4}$ \\ ${ }^{1}$ Department of Neurosurgery, Ewha Womans University Seoul Hospital, Ewha Womans University College of Medicine, Seoul, Korea \\ ${ }^{2}$ Department of Neurosurgery, International St. Mary's Hospital, Catholic Kwandong University College of Medicine, Incheon, Korea \\ ${ }^{3}$ Department of Radiology, Ajou University Hospital, Ajou University College of Medicine, Suwon, Korea \\ ${ }^{4}$ Department of Radiology, Gangnam Severance Hospital, Yonsei University College of Medicine, Seoul, Korea
}

Purpose: In Korea, endovascular treatment (EVT) for intracranial aneurysms (IAs) has increased steadily. We conducted a nationwide survey to evaluate the current status of EVT for IAs and to identify treatment preference in the real world.

Materials and Methods: A Google online survey was distributed to representative clinicians at hospitals treating IAs, where members of Korean Society of Interventional Neuroradiology (KSIN). The data was collected from October 2017 to December 2017. The responding hospitals were divided into 2 groups (tertiary and non-tertiary hospitals). And variable factors involved in decision making for treatment were evaluated.

Results: In total, 73 hospitals (tertiary: 37, non-tertiary: 36) responded to the survey. Most hospitals that responded had over 100 cases of diagnostic angiography (93\%) and over 50 cases of EVT for IAs (74\%) performed in 2016. The proportion of EVT for ruptured aneurysms in non-tertiary hospitals was significantly higher than tertiary hospitals (49\% vs. 9\%). The proportion of EVT for unruptured aneurysms at non-tertiary hospitals was significantly higher than tertiary hospitals (66\% vs. 44\%). Most physicians tended to make decision for treatment on location, shape, and size of unruptured IAs and patients' age, more than the results from previous clinical trials for unruptured IAs. Although EVT was preferred for older patients (age $>70$ ) with unruptured IAs (99\%), surgical clipping was still considered as the first treatment of choice for younger patients (age 30 to 50 years) at considerable rates (56\%). Over two-thirds of respondents preferred surgical clipping for middle cerebral artery aneurysms, while EVT was preferred initially at other locations.

Conclusion: This nationwide survey showed that EVT is considered as the first treatment modality for IAs and there is a discrepancy between current guidelines and real-world practice for decision making of treatment options.

Key Words: Aneurys; Treatment; Surveys; Hospitals; Korea

\section{Correspondence to: Sang Hyun Suh, MD, PhD Department of Radiology, Gangnam Severance Hospital, Yonsei University College of Medicine, 211 Eonju-ro, Gangnam-gu, Seoul 06273, Korea Tel: $+82-2-2019-3510$ \\ Fax: +82-2-3462-5472 \\ E-mail: suhsh11@yuhs.ac}

Received: July 3, 2019

Revised: August 10, 2019

Accepted: December 2, 2019
Copyright $\odot 2020$ Korean Society of Interventional Neuroradiology This is an Open Access article distributed under the terms of the Creative Commons Attribution Non-Commercial License (http://creativecommons.org/licenses/by-nc/3.0) which permits unrestricted non-commercial use, distribution, and reproduction in any medium, provided the original work is properly cited. 


\section{INTRODUCTION}

After the introduction of the Guglielmi detachable coils in 1993, endovascular treatment (EVT) has consistently contributed to the treatment of intracranial aneurysms (IAs) in Korea, and EVT has become a more common therapy for IAs than microsurgical clipping since 2012.' Recently, various data sets were used to report the current status of neurointervention in Korea, including the status of neurointerventional radiographic equipment and professionals and IA treatment using the national health insurance. ${ }^{1-5}$ However, there were no studies reflecting clinical practice and treatment strategies for EVT of IAs from the real world. We tried to collect and evaluate the data directly from neurointerventionists in Korea to understand the decision-making process for treatment of IAs as well as the clinical status of IA treatment.

\section{MATERIALS AND METHODS}

The survey was designed to collect data from a questionnaire written in Korean. Among hospitals where members of Korean Society of Interventional Neuroradiology (KSIN) work at, the questionnaire was electrically sent to a representative clinician at each hospital where EVT was available in Korea. The representative clinician was asked to submit their hospital data. This survey was carried out from October 2017 to December 2017. A Google survey was used to distribute this survey questionnaire and receive their responses.

The concerned data was composed of 29 single or multiple-choice questions about general information of each hospital, current overview, number of doctors involved in neurointervention, specialties of clinician, treatment strategy between ruptured versus unruptured IAs, and other factors that had an influence on deciding the treatment option. The factors include age, sex of the patient, location, size, shape of aneurysm, and other personal medical history.
Korea has a 3-level hierarchy of hospitals, which were dichotomized into 2 categories, tertiary and non-tertiary hospitals, including primary and secondary hospitals. The collected data was first divided according to the type of hospital, and the factors related to decision making procedures were reviewed.

These responses were analyzed using the chi-square or Fischer's exact test with IBM SPSS Statistics for Windows (version 23.0; IBM Corp., Armonk, NY, USA).

\section{RESULTS}

A total of 73 hospitals responded to the survey, including 37 tertiary hospitals and 36 non-tertiary hospitals. The presented percentage of data of each category was analyzed for each total data. Most of the responding hospitals were located in Seoul, Gyeong-Gi Province, and Gyeongsang Provinces $(61 / 73 ; 84 \%)$.

\section{Characteristics of respondents and responding hos- pitals}

Based on the clinical specialty of the respondents, radiologists mostly responded in tertiary hospitals (26/37; 70\%), and neurosurgeons mostly responded in non-tertiary hospitals $(21 / 36 ; 58 \%)$. The majority of total responders had over 5 years of personal independent experience in endovascular procedures $(59 / 73 ; 81 \%)$. In $88 \%$ of hospitals $(64 / 73)$, more than 2 neurointerventionists were working together. The proportion of neurosurgeons was higher in non-tertiary than in tertiary hospitals (Fig. 1).

In most responding hospitals, diagnostic cerebral angiography was performed in over 100 cases in 2016 (68/73; 93\%). Moreover, more than 1,000 cases of diagnostic cerebral angiography were performed in 7 tertiary hospitals and 1 non-tertiary hospital $(8 / 73 ; 11 \%)$. Almost all hospitals ran 24-hour on-call schedule duty (72/73; 99\%) and an interdis-
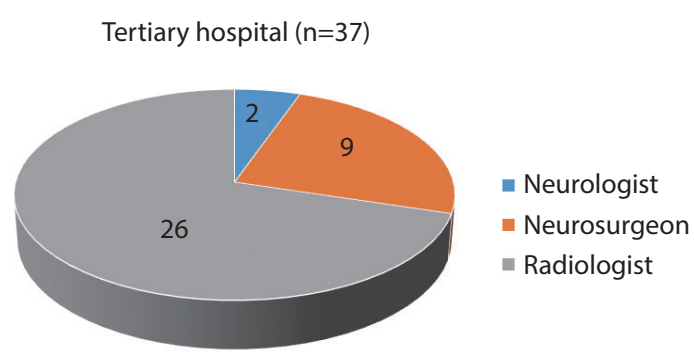

Non-tertiary hospital $(n=36)$

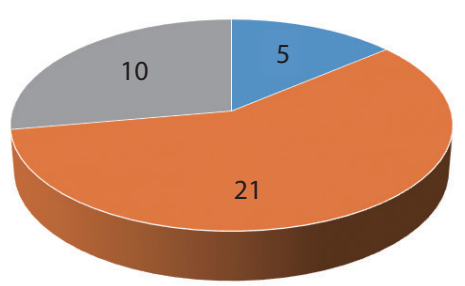

Fig. 1. The number of specialists who work as interventionists in tertiary and non-tertiary hospitals. 
ciplinary consultation system (68/73; 93\%). The fellowship program was offered in 36\% (27/73) of responding hospitals.

Specialized stroke unit and neurosurgical care units were operated in 69\% (50/73) and 30\% (22/73) of hospitals, respectively. Most responding hospitals (68/73) operated biplane digital subtraction angiography, computed tomography, and magnetic resonance imaging.

\section{General information about IA treatment}

In this category, a total of 70 hospitals (tertiary: 35, non-tertiary: 35) answered the questionnaire. In 2016, 91\% (32/35) of tertiary hospitals had treated more than 100 IAs, compared to $66 \%(23 / 35)$ of responding non-tertiary hospitals. Moreover, 8 tertiary hospitals treated over 300 cases of an aneurysm in the same year (Fig. 2). For EVT of aneurysms, a majority of tertiary hospitals operated over 50 cases of an aneurysm (33/35; 94\%), while 51\% (18/35) of non-tertiary hospitals operated over 50 cases.

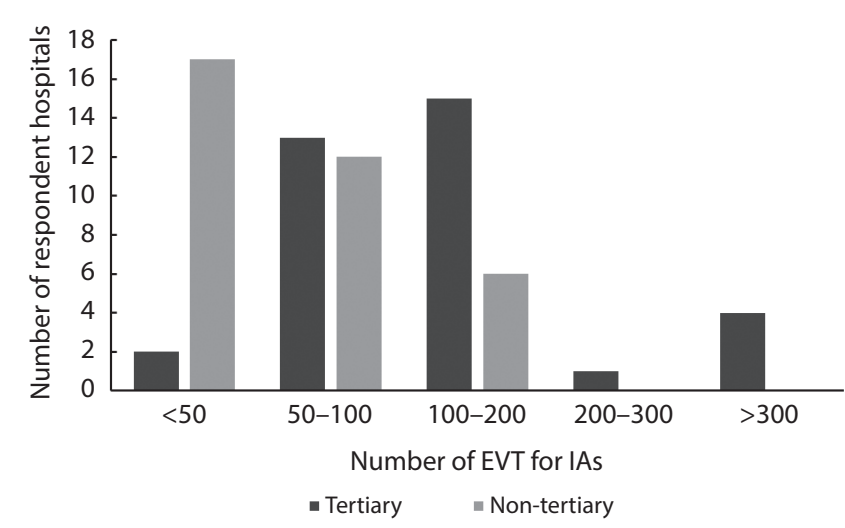

Fig. 2. EVT of IAs in 2016 according to the type of hospital. EVT, endovascular treatment; IAs, intracranial aneurysms.

Table 1. Factors that physicians considered in decision making to treat ruptured aneurysms with EVT

\begin{tabular}{lc}
\hline Factor & Value \\
\hline Location of aneurysm & $63(94)$ \\
Size of aneurysm and aspect ratio & $49(73)$ \\
Age & $46(69)$ \\
Hunt and Hess/WFNS grade & $35(52)$ \\
Sex & $5(7)$ \\
\hline
\end{tabular}

Values are presented as number (\%). Multiple responses were possible. The percentage of each factor is the proportion out of a total of 66 responders.

EVT, endovascular treatment; WFNS, World Federation of Neurosurgical Societies.

\section{Treatment for ruptured IAs}

In this category, 68 hospitals (tertiary: 33, non-tertiary: 35) answered. The proportion of EVT for ruptured aneurysms in non-tertiary hospitals (17/35, 49\%) was significantly higher than the proportion in tertiary hospitals (3/33, 9\%). In 65\% (44/68) of all responding hospitals, over $50 \%$ of ruptured aneurysms were treated with EVT.

The location (94\%), size of aneurysm and aspect ratio (73\%), and age (69\%) mostly affected decision making to treat the ruptured aneurysm with EVT (Table 1).

\section{Treatment for unruptured IAs}

\section{Percentage of EVT for unruptured IAs}

In this category, 67 hospitals (tertiary: 32, non-tertiary: 35) answered. In 2016, most of responding hospitals (85\%) tend to treat more unruptured IAs than ruptured IAs (65\%) and the proportion of EVT for unruptured aneurysms in non-tertiary hospitals $(23 / 35,66 \%)$ was higher than that in tertiary hospitals $(14 / 32,44 \%)$.

\section{Criteria for treatment of unruptured IAs}

In this category, 67 hospitals (tertiary: 32, non-tertiary: 35) answered. Overall, 61\% (41/67) of respondents had no criterion for unruptured IA treatment, and the remaining considered some criteria, including International Study of Unruptured Intracranial Aneurysms (ISUIA) study, ${ }^{6}$ Unruptured Cerebral Aneurysms Study of Japan, ${ }^{7}$ or PHASES score. ${ }^{8}$

In Table 2, decisions whether to treat unruptured aneurysms were based on the size (91\%), shape (91\%), and location (70\%) of aneurysms. Additionally, age (61\%) and personal

Table 2. Factors that physicians considered in decision making to treat unruptured aneurysms with EVT

\begin{tabular}{lc}
\hline Factor & Value \\
\hline Size of aneurysm & $61(91)$ \\
Shape of aneurysm & $61(91)$ \\
Location of aneurysm & $47(70)$ \\
Age & $42(64)$ \\
Family or personal history of SAH & $28(42)$ \\
Related risk factors (smoking, hypertension, etc.) & $10(15)$ \\
\hline Sex & $3(5)$ \\
\hline
\end{tabular}

Values are presented as number (\%). Multiple responses were possible. The percentage of each factor is the proportion out of a total of 67 responders.

EVT, endovascular treatment; SAH, subarachnoid hemorrhage. 
or family history of subarachnoid hemorrhage (SAH) (42\%) were factors.

\section{Decision-making based on patient's age}

In this category, 67 hospitals (tertiary: 32, non-tertiary: 35) responded. The majority of respondents preferred EVT for unruptured aneurysms with increasing patients' age over 60 (60-70 years, 95\%; over 70 years, 99\%), and half of the respondents still considered surgical clipping for younger patients at considerable rates (under 30 years, 52\%; 30-50 years, 56\%) (Table 3).

In addition, treatment preference of unruptured aneurysms at age 50 to 60 differed between tertiary (EVT 65\%) and non-tertiary hospitals (EVT 79\%), showing no statistical significance $(P=0.2)$.

\section{Decision-making based on size of IAs}

Regardless of the location of aneurysm, respondents (77$100 \%)$ tended to treat most aneurysms over $5 \mathrm{~mm}$ in size (Table 4). Moreover, 81\% (59/63) and 73\% (53/63) of respondents indicated treatment for anterior communicating artery (ACOM) aneurysms and posterior communicating artery
(PCOM) aneurysms of 3 to $5 \mathrm{~mm}$ in size.

\section{Decision-making based on location of IAs}

In this category, the number of responding hospitals differed for each question from 65 to 67. Regarding the location of aneurysm, most respondents treated wide-necked internal carotid artery aneurysms preferentially with stent-assisted coil embolization $(63 / 67,94 \%)$ rather than coil embolization without a stent (16/67, 24\%, Table 5). On the contrary, ACOM aneurysms and PCOM aneurysms were treated preferentially with coil embolization without stent (57/66, 86\%; 54/67, 81\%) rather than stent-assisted coil embolization (26/66, 39.4\%; 39/67, 58\%). Most respondents preferred coil embolization for vertebrobasilar aneurysms and basilar apex aneurysms. Surgical clipping still remained the preferred treatment strategy for middle cerebral artery aneurysms (46/66, 70\%). However, there was a difference in the proportion of preference for surgical clipping between tertiary hospitals (27/33, 82\%) and non-tertiary hospitals (19/33, 58\%).

Table 3. Selection of surgical treatment of unruptured IAs according to age of patient in total respondent hospitals

\begin{tabular}{lccccc} 
& \multicolumn{4}{c}{ Age $(\mathrm{n}=65)$ (years) } \\
\cline { 2 - 6 } & $<30$ & $30-50$ & $50-60$ & $60-70$ & $>70$ \\
\hline Treatment & & & & & \\
$\quad$ Surgical clipping & $33(52.4)$ & $33(52.4)$ & $18(28.1)$ & $3(4.6)$ & $1(1.5)$ \\
EVT & $30(47.6)$ & $28(44.4)$ & $46(71.9)$ & $62(95.4)$ & $64(98.5)$ \\
\hline
\end{tabular}

Values are presented as number (\%).

IAs, intracranial aneurysms; EVT, endovascular treatment.

Table 4. Treatment decision rate of clinicians according to the size and location of unruptured IAs in total responding hospitals

\begin{tabular}{|c|c|c|c|c|c|c|c|c|c|c|}
\hline & \multicolumn{10}{|c|}{ Size of UIAs (mm) } \\
\hline & \multicolumn{2}{|r|}{$<3$} & \multicolumn{2}{|r|}{$3-5$} & \multicolumn{2}{|c|}{$5-7$} & \multicolumn{2}{|c|}{$7-10$} & \multicolumn{2}{|r|}{$>10$} \\
\hline & $\begin{array}{l}\text { Tertiary } \\
(n=16)\end{array}$ & $\begin{array}{c}\text { Non-tertiary } \\
(n=18)\end{array}$ & $\begin{array}{l}\text { Tertiary } \\
(n=29)\end{array}$ & $\begin{array}{c}\text { Non-tertiary } \\
(n=34)\end{array}$ & $\begin{array}{c}\text { Tertiary } \\
(n=31)\end{array}$ & $\begin{array}{c}\text { Non-tertiary } \\
(n=30)\end{array}$ & $\begin{array}{l}\text { Tertiary } \\
(n=30)\end{array}$ & $\begin{array}{c}\text { Non-tertiary } \\
(n=27)\end{array}$ & $\begin{array}{l}\text { Tertiary } \\
(n=29)\end{array}$ & $\begin{array}{c}\text { Non-tertiary } \\
(n=26)\end{array}$ \\
\hline ICA & $1(6)$ & $2(11)$ & $12(41)$ & $14(41)$ & $29(94)$ & $23(77)$ & $29(97)$ & $24(89)$ & $29(100)$ & $25(96)$ \\
\hline MCA & $6(38)$ & $10(56)$ & $23(79)$ & $20(59)$ & $30(97)$ & $29(97)$ & $29(97)$ & $25(93)$ & $28(97)$ & $24(92)$ \\
\hline ACOM & $14(88)$ & $14(78)$ & $29(100)$ & $30(88)$ & $29(94)$ & $26(87)$ & $29(97)$ & $25(93)$ & $29(100)$ & $24(92)$ \\
\hline PCOM & $12(75)$ & $10(56)$ & 27 (93) & $26(77)$ & 27 (87) & $25(83)$ & $27(90)$ & $27(100)$ & 27 (93) & 25 (96) \\
\hline VB & $12(75)$ & $6(33)$ & $22(76)$ & $25(74)$ & $28(90)$ & $23(77)$ & $27(90)$ & $22(82)$ & 27 (93) & $23(89)$ \\
\hline
\end{tabular}

Values are presented as number (\%).

IAs, intracranial aneurysms; UIAs, unruptured intracranial aneurysms; ICA, internal carotid artery; MCA, middle cerebral artery; ACOM, anterior communicating artery; PCOM, posterior communicating artery; VB, vertebrobasilar artery. 
Table 5. Decision making of treatment modality based on location of unruptured IAs

\begin{tabular}{|c|c|c|c|c|c|c|}
\hline & \multicolumn{6}{|c|}{ Location of UIAs } \\
\hline & $\begin{array}{c}\text { Wide-necked } \\
\text { internal carotid } \\
\text { artery }\end{array}$ & $\begin{array}{c}\text { Anterior } \\
\text { communicating } \\
\text { artery }\end{array}$ & $\begin{array}{c}\text { Posterior } \\
\text { communicating } \\
\text { artery }\end{array}$ & $\begin{array}{c}\text { Vertebro-Basilar } \\
\text { artery }\end{array}$ & $\begin{array}{c}\text { Basilar artery } \\
\text { apex }\end{array}$ & $\begin{array}{c}\text { Middle cerebral } \\
\text { artery }\end{array}$ \\
\hline \multicolumn{7}{|l|}{ Treatment option } \\
\hline Flow diverter & $11(16.4)$ & $1(1.5)$ & $1(1.5)$ & $8(12.3)$ & $2(3.0)$ & $1(1.5)$ \\
\hline Balloon-assisted coiling & $11(16.4)$ & $2(3.0)$ & $5(7.5)$ & $3(4.6)$ & $5(7.6)$ & $1(1.5)$ \\
\hline Stent-assisted coiling & $63(94.0)$ & $28(42.4)$ & $39(58.2)$ & $55(84.6)$ & $55(83.3)$ & $19(28.8)$ \\
\hline Coiling without stent & $16(23.9)$ & $57(86.4)$ & $54(80.6)$ & $49(75.4)$ & $56(84.8)$ & $39(59.1)$ \\
\hline Surgical clipping & $7(10.4)$ & $30(45.5)$ & $21(31.3)$ & $3(4.6)$ & $1(1.5)$ & $46(69.7)$ \\
\hline Observation & $10(16.9)$ & $1(1.5)$ & $1(1.5)$ & $2(3.1)$ & $2(3.0)$ & $3(4.5)$ \\
\hline
\end{tabular}

Values are presented as number (\%).

IAs, intracranial aneurysms; UIAs, unruptured intracranial aneurysms.

\section{DISCUSSION}

This study demonstrates that EVT for IAs is the preferred treatment strategy in Korea. This is the first recent survey to describe and quantify treatment strategies of IAs by using data from 73 domestic hospitals that mainly treat most IAs in real clinical situations. The proportion of EVT in non-tertiary hospitals tended to be higher than in tertiary hospitals for both ruptured and unruptured IAs. In Korea, unruptured IAs that are 3 to $5 \mathrm{~mm}$ and located on the ACOM, PCOM, and vertebrobasilar artery tended to be treated with clipping or coiling.

After publication of the International Subarachnoid Aneurysm Trial $^{9}$ and the ISUIA, ${ }^{6}$ the proportion of EVT for IAs has consistently increased. In Korea, coil embolization has been the treatment of choice over surgical clipping in the management of IAs based on Health Insurance Review \& Assessment Service (HIRA) data since 2012.' They reported that EVT is considered as the first treatment modality in 64\% of ruptured IAs and $85.1 \%$ of unruptured IAs. Interestingly, the proportion of EVT in non-tertiary hospitals tended to be higher than that in tertiary hospitals for ruptured (EVT/ total treatment of ruptured aneurysms $>70 \%$; $49 \%$ vs. $9 \%$, respectively) and unruptured aneurysms (EVT/total treatment of unruptured aneurysms $>70 \% ; 66 \%$ vs. $44 \%$, respectively), even though there was no difference in the factors which affected treatment strategy.

Fargen et al. ${ }^{10}$ summarized that most physicians make decisions to treat unruptured aneurysms based on the result of the ISUIA study. ${ }^{6}$ However, most respondents in this study considered size, location, and shape of aneurysms as main factors for treatment strategy and reported using none of the data of ISUIA study, ${ }^{6}$ UCAS Japan, ${ }^{7}$ or PHASES score ${ }^{8}$ to make decisions to treat unruptured aneurysms (63\%). Even though the authorized guidelines in Korea ${ }^{11,12}$ recommend treatment of unruptured IAs over $5 \mathrm{~mm}$ or under $5 \mathrm{~mm}$ with risk factors for rupture, considerable cases of unruptured IAs that are 3 to $5 \mathrm{~mm}$ were treated in the real world. In Korea, the majority of respondents treated unruptured IAs that are 3 to $5 \mathrm{~mm}$ and located on ACOM, PCOM, and vertebrobasilar artery. Although several studies showed considerable rupture risk of unruptured IAs under 5 mm, 13,14 real-world practice needs to be supported by clinical evidence.

As endovascular devices and techniques have advanced and clinical outcomes of EVT have improved, most respondents preferred EVT as first treatment modality for unruptured IAs except middle cerebral artery (MCA) bifurcation aneurysms. For MCA bifurcation aneurysms, as was the result of survey in the United States, ${ }^{10}$ two-thirds of respondents considered surgical clipping as the first treatment modality. In the cases of wide-necked IAs, while most physicians recommended flow diverters in the United States, ${ }^{10}$ a majority of respondents preferred stent-assisted coil embolization $(63 / 67,94 \%)$ rather than flow diverters $(11 / 67,16 \%)$ in this study. This discrepancy might result from more strict criteria of the HIRA, where the use of flow diverters is restricted for unruptured IAs over $15 \mathrm{~mm}$ without concomitant use of detachable coils. The modification of treatment criteria for unruptured aneurysms might be necessary for realistic practice and developmental trends in EVT.

The current study has some limitations. First, this survey represented personal preference for treatment strategies of 
each respondent. Although decision-making for treatment of IAs might be a complicated and multi-factorial process, respondents answered concise and categorized questionnaires. Therefore, cautious application of the results might be necessary for treatment of individual IAs. Second, as the result was derived from responses of the survey, inherent biases including sampling error, selection bias, and recall bias might occur. While the survey was not a complete enumeration, a considerable response rate ( 73 of $120 \mathrm{KSIN}$ membership hospitals) could reduce sampling error. In addition, these results might be supported by the HIRA data, which covers clinical practice nationwide.

\section{CONCLUSION}

This study is the first nation-wide survey in Korea to demonstrate the current status of EVT and preferred treatment strategies for IAs in the real world. Most respondents recommended EVT as a first treatment modality for both ruptured and unruptured IAs, and the size, shape, and location of the aneurysm were major factors that they considered. Most physicians did not use the sole result of previous clinical trials to decide treatment of unruptured aneurysms, but they considered location, shape, and size of an aneurysm and patients' age together. As there is a discrepancy between current guidelines and real-world practice for decision making of treatment options, consensual approaches revealing real clinical situations for performing EVT in proper indications should be suggested.

\section{Fund}

This study was supported by the Korean Society of Interventional Neuroradiology (KSIN) research grant 2017.

\section{Ethics Statement}

This study was approved by the Institutional Review Board of Gangnam Severance Hospital.

\section{Conflicts of Interest}

The authors have no conflicts to disclose.

\section{REFERENCES}

1. Suh SH. The annual trends between neurointerventional and neurosurgical procedures in Korea: analysis using HIRA data from 2010 to 2016. Neurointervention 2017;12:77-82

2. Lee $W K$, Oh $C W$, Lee $H$, Lee $K S$, Park $H$. Factors influencing the incidence and treatment of intracranial aneurysm and subarachnoid hemorrhage: time trends and socioeconomic disparities under an universal healthcare system. J Neurointerv Surg 2019;11:159-165

3. Lee YM, Hwang SM, Kim EH, Lee DG, Shim JH, Suh DC. Current status of neurointerventional activities in Korea. Neurointervention 2013;8:65-67

4. Ryu CW, Hwang SK, Jung HD, Park YS, Park MH, Kim HJ. The present status of neurointerventional angiographic systems and workers in Korea. Neurointervention 2009;4:116-124

5. Kim JE, Lim DJ, Hong CK, Joo SP, Yoon SM, Kim BT. Treatment of unruptured intracranial aneurysms in South Korea in 2006 : a nationwide multicenter survey from the korean society of cerebrovascular surgery. J Korean Neurosurg Soc 2010;47:112-118

6. International Study of Unruptured Intracranial Aneurysms Investigators. Unruptured intracranial aneurysms--risk of rupture and risks of surgical intervention. N Engl J Med 1998;339:17251733

7. UCAS Japan Investigators, Morita A, Kirino T, Hashi K, Aoki N, Fukuhara S, et al. The natural course of unruptured cerebral aneurysms in a Japanese cohort. N Engl J Med 2012;366:2474-2482

8. Greving JP, Wermer MJ, Brown RD Jr, Morita A, Juvela S, Yonekura $M$, et al. Development of the PHASES score for prediction of risk of rupture of intracranial aneurysms: a pooled analysis of six prospective cohort studies. Lancet Neurol 2014;13:59-66

9. Molyneux A, Kerr R, Stratton I, Sandercock P, Clarke M, Shrimpton J, et al. International Subarachnoid Aneurysm Trial (ISAT) of neurosurgical clipping versus endovascular coiling in 2143 patients with ruptured intracranial aneurysms: a randomised trial. Lancet 2002;360:1267-1274

10. Fargen KM, Soriano-Baron HE, Rushing JT, Mack W, Mocco J, Albuquerque $F$, et al. A survey of intracranial aneurysm treatment practices among United States physicians. J Neurointerv Surg 2018;10:44-49

11. Jeong HW, Seo JH, Kim ST, Jung CK, Suh SI. Clinical practice guideline for the management of intracranial aneurysms. Neurointervention 2014;9:63-71

12. Seo DH, Kang HS, Kim DW, Park SQ, Song Y, Sheen SH, et al. Guidelines for the management of unruptured intracranial aneurysm. Korean J Cerebrovasc Surg 2011;13:279-290 
13. Bender MT, Wendt $H$, Monarch T, Beaty N, Lin LM, Huang J, et al. Small aneurysms account for the majority and increasing percentage of aneurysmal subarachnoid hemorrhage: a 25-year, single institution study. Neurosurgery 2018;83:692-699
14. Sonobe M, Yamazaki T, Yonekura M, Kikuchi H. Small unruptured intracranial aneurysm verification study: SUAVe study, Japan. Stroke 2010;41:1969-1977 